\title{
Response of Prefrontal Cortex to Executive Function Tasks in Early Childhood: An Exploratory Case Study for Childcare
}

\author{
Nobuki Watanabe $^{1}$ \\ ${ }^{1}$ School of Education, Kwansei Gakuin University, Japan \\ Correspondence: Nobuki Watanabe, School of Education, Kwansei Gakuin University, 7-54, Okadayama, \\ Nishinomiya, Hyogo, 662-0827, Japan.
}

Received: May 7, 2021

Accepted: May 31, 2021

Online Published: June 7, 2021

doi:10.5539/ijps.v13n3p12

URL: https://doi.org/10.5539/ijps.v13n3p12

\begin{abstract}
Executive function (EF) development is remarkable in early childhood. EF is an ability that provides a foundation for future success; accordingly, supporting children during their early childhood is crucial. So far, there have been many findings on EF in early childhood based on behavioral observation. This exploratory case study examines the measurement of prefrontal cortex activity during the performance of EF tasks using functional near-infrared spectroscopy (fNIRS). This study aims to explore the following hypothesis. (1) The prefrontal cortex during early childhood becomes more active as a task becomes more difficult. (2) However, brain activity decreases and stabilizes as time progresses. (3) The evaluation can be easily measured with two-channel fNIRS. Experimental results showed that the preschooler and the child in lower elementary school displayed high levels of brain activity in the order of increasing difficulty in terms of behavioral indicators. Moreover, the preschooler showed higher levels of reaction than the child in lower elementary school. This result is useful and will broaden the perspectives of scholars in the fields of psychology, pedagogy, and neuroscience, those involved in child support.
\end{abstract}

Keywords: executive function, prefrontal cortex, functional near-infrared spectroscopy, subcomponent, exploratory case study

\section{Introduction}

Executive function (EF) is an advanced cognitive control process (Moriguchi \& Hiraki, 2013; Moriguchi, 2015) and is involved in various aspects of cognitive development (Anderson, Storfer-Isser, Taylor, Rosen, \& Redline, 2009). With regard to the structure, its development has a strong genetic influence (Friedman et al., 2008), where the child-rearing environment is an important factor (Anderson, Storfer-Isser, Taylor, Rosen, \& Redline, 2009). Previous studies have found that inhibition, cognitive shifting, and working memory are the subcomponents of EF in adults (Miyake et al., 2000; Moriguchi \& Hiraki, 2013). Furthermore, findings from previous studies indicated that the sub-elements can be classified as hot (emotive) and cool (cognitive) (Zelazo \& Carlson, 2012). In addition, EF is related to the function of the prefrontal cortex (Burgess \& Stuss, 2017; Stuss \& Alexander, 2000). Moreover, the developmental sprouting of EFs is seen in infancy (infants: 0 years old, toddlers: 1-2 years old), and it develops markedly in early childhood (preschool children: 3-6 years old), with gradual development continuing from childhood (lower elementary school children: 7-9 years old, higher elementary school children: 10-12 years old) to adulthood (Moriguchi, 2015, 2019).

Tasks often used as indicators of each component have clearly provided behavioral evidence of the subcomponents for infants. The following tests are used as indexes: Stroop's task of inhibition for the cool task (Stroop, 1935), the Dimensional Change Card Sort (DCCS) for cognitive shifting (Zelazo, 2006), Wechsler Intelligence Scale for Children (WISC-IV) Working Memory Index (WMI) task (digit span, letter-number sequencing, and arithmetic) (Wechsler, 2003) for working memory, and Marshmallow test for the hot task (Mischel, 2014).

Conversely, although anatomical knowledge about prefrontal cortex activity related to EF in infants exists (Adleman et al., 2002; Li, Lu, D’Argembeau, Ng, \& Bechara, 2010; Mischel, 2014; Moriguchi \& Hiraki, 2009; Schroeter, Zysset, Wahl, \& von Cramon, 2004), neurophysiological evidence of EF (and each subcomponent) has been accumulating (Moriguchi \& Hiraki, 2013). Non-invasive, continuous, and simple functional near-infrared spectroscopy (fNIRS) (Hoshi, 2005; Igawa et al., 2001; Villringer, Planck, Hock, Schleinkofer, \& 
Dirnagl, 1993) is largely used to collect evidence of EF in infants due to its flexibility and low-cost (Moriguchi \& Hiraki, 2013).

Results to date suggest that the prefrontal cortex may be activated when demand increases in infants and that its activity may decrease with age (Moriguchi \& Hiraki, 2013). Many issues have been related to DCCS in terms of research on EF index tasks using fNIRS in young children (Buss \& Spencer, 2018; Kerr-German \& Buss, 2020; Moriguchi \& Hiraki, 2013). From the above, these can be predicted theoretically/conceptually. However, research on other tasks and the relationship between such issues remain unknown. The clarification of this issue would constitute a significant contribution to support for the promotion of EFs because there has been no unified indicator of EF. Moreover, such a clarification would indicate that scientific evidence can be easily obtained for children, for whom researchers have difficulties in obtaining behavioral evidence. At present, studies have found that subcomponents of $\mathrm{EF}$ are present in infants with abundant evidence collected for each behavior. Furthermore, knowledge of the effectiveness of fNIRS measurement exists. Therefore, the purpose of this paper is to explore the following hypothesis.

For EF in early childhood, (1) this study infers that assessing the characteristics of activity or reactions in the prefrontal cortex is possible by measuring the brain activity of the representative index task of each subcomponent using fNIRS. (2) Brain activity in the prefrontal cortex during early childhood becomes more active as the task becomes more difficult. (3) However, its activity decreases and stabilizes as the time progresses. (4) This kind of evaluation can be easily evaluated with two-channel fNIRS.

Therefore, this exploratory case study used fNIRS to measure brain activity in the prefrontal cortex during the performance of representative index tasks for each subcomponent. The subjects are a preschooler aged 4 years (between 4;7 and 4;8) and his older sister aged 8 years (between 8;6 and 8;7), who is in lower elementary school; siblings were chosen for their similar genetic and parenting environment. The study sought to verify whether the characteristics and trends of brain activity in the prefrontal cortex can be assessed through cross-sectional measurements for developing a novel hypothesis.

\section{Materials and Methods}

\subsection{Study Design}

Each task (cool and inhibition: Stroop's task; cognitive shift: DCCS; working memory: WISC-IV; hot: Marshmallow) is representative of the sub-constituent function of EF and were carried out on the subjects. Brain activity in the prefrontal cortex during each task in relation to EF was measured using fNIRS, and behavioral results were measured. A two-channel fNIRS device was used, with emphasis on functionality. The measured value was left and right total-Hb (hemoglobin) (mMmm). A two-channel fNIRS instrument Hot-2000 (NeU; Japan; cost: 198,000 yen) was used in this study. It uses the light of wavelength (approximately $800 \mathrm{~nm}$ ). A detector is positioned about $1 \mathrm{~cm}$ (for acquiring scalp blood flow information that has passed through the shallow scalp) and $3 \mathrm{~cm}$ (for acquiring scalp blood flow information that has passed through the shallow scalp) from the light irradiation. Brain activation is estimated from the rate. The total-Hb change (left/right subtracted) is each brain activity index (spike noise is removed; the signal at SD distance of $3 \mathrm{~cm}$ minus the signal at SD distance of $1 \mathrm{~cm}$ is subtracted at a constant rate). In general, the frequency of measurement was once per day for each task. At this point, measurement was evaluated only when the physical condition and mood of the children were sufficiently excellent. For this reason, this case study is considered exploratory.

\subsection{Prefrontal Cortex Measurement Device (fNIRS)}

Brain activity in fNIRS was measured as total hemoglobin (total-HB). Currently, one of the simple and low-cost fNIRS instruments is the two-channel type. Notably, this instrument can measure total-Hb of left and right sides of the prefrontal cortex, which have been shown to be associated with the local activation of the brain (Haida, 2002; Igawa et al., 2001).

\subsection{Target}

The subjects are a four-year-old boy, who is considered to have a significant EF development period (i.e., in early childhood), and his eight-year-old older sister, whose age and cognitive development period were sufficiently advanced (i.e., in lower childhood). They were labeled as Subjects A and B for easy reference in other parts of the manuscript (i.e., Subject A for the 4-year-old boy and Subject B for the 8-year-old older sister). Siblings were employed for the study to ensure nearly similar genetic and child-rearing environments. The parents were briefed in person and in writing about the process of the research. Their parents were assured of anonymity about the subjects. Written informed consent was obtained from their parents before the research. 
This study has been approved by my university's research ethics committee. This study has been conducted in accordance with the Code of Ethics of the World Medical Association (Declaration of Helsinki).

\subsection{Method/Brain Activity Measurement}

Each task was conducted thrice or more as appropriate. Brain activity was measured when the task was performed. Data were excluded when nonstandard or unobtainable. In addition, the brain activity device was worn for several minutes before a task, and a relaxing time was provided while seated on a chair. If the total- $\mathrm{Hb}$ on the left and right was less than $0.1(\mathrm{mMmm})$ within 10 seconds (s) during relaxation, it was recorded as a rest period. Data were excluded in the absence of a rest period.

\subsection{Protocol/Subcomponent Indicator Task: Cognitive Element (Cool)}

\subsubsection{Inhibition and Stroop's Task (Color-word Task)}

The task is a general color-word test, which consisted of reading character strings representing color names and was deemed appropriate for the subject, who can read the characters. As a non-interference task, the researcher read a character string representing the same color as the color name (i.e., "green" text is used to represent the color green). As an interference task, a character string in one color was used to represent a different color (i.e., red text is used to represent the color green). One text is displayed on one PowerPoint page.

In this task, rest time and an explanation of the non-interfering task were given; next a non-interfering task was conducted. This was then followed by rest time and an explanation of interfering task, and finally an interfering task was executed; these processes were carried out consecutively. First, a rest period of $30 \mathrm{~s}$ and a task explanation period were provided. Subsequently, the non-interference task was conducted for $60 \mathrm{~s}$, and another rest period of $15 \mathrm{~s}$ was taken before the task. Then, the interference task was carried out for $60 \mathrm{~s}$.

A blank sheet was displayed for $1 \mathrm{~s}$ after displaying a character string for $3 \mathrm{~s}$. A total of 15 questions were asked in each task. For brain measurement, the interference task is considered to elicit significant EF.

\subsubsection{Cognitive Shifting: DCCS Task (Combined Pre- and Post-switch Task)}

A general protocol was used. The task was conducted following the procedures outlined in Zelazo (2006). The researcher prepared two dimensions in the pre-switch and post-switch tasks; namely, color and shape. Specifically, the colors used were red and blue, and the shapes were a car and a star. Target cards, such as a "blue car" and a "red star" were presented in the study. The classification card has the colors reversed, i.e., classification card was a "blue car" and a "red star." The pre-switch task was classified using a one dimension and the post-switch task was classified using the other dimension. The case of classifying by color was called a color game and that by shape was called a shape game. Three "blue car" cards and three "red star" cards were used in each task. The same card was not presented three times in a row. For the mixture task (border task), three "blue car" cards and three "red star" cards were used in each task. Moreover, three "blue car" cards were added with black outside the frame of the cards in addition to three "red star" cards with black outside the frame of the cards. There were 12 cards in total. In the task, when a card with black outside the frame was presented, the target needed to adopt the pre-determined rules (shape game or color game). When a card without black outside the frame was presented, the target needed to adopt the pre-determined rules. The rule was a different game (shape game or color game) from the previous rule. The brain activity was measured in both the post-switch and mixture tasks. The measurement times for each task differed. In this study, the set of the other cards were used too. The set was as follows. Target cards were a yellow triangle and purple beetle, and classification cards were purple triangle and yellow beetle. In the study, the pre-switch task was performed twice in the color game and once in the shape game.

\subsubsection{Working Memory: WISC-IV-WMI Task}

WISC-IV tasks differ according to age. This task was conducted according to the general manual. That is, the task was carried out following the procedures outlined in Wechsler (2003). For WMI tasks, digit span, letter-number sequencing, and arithmetic were performed in this order. Following the manual, the task was completed when the condition of "cancel" was satisfied. In the same manner, the time spent for implementing the task differed. Brain activity was measured during implementation.

\subsection{Protocol/Subcomponent Indicator Task: Emotive Elements}

\subsubsection{Marshmallow Test}

The test was implemented using a general protocol. That is, the task was conducted following the procedures outlined in Mischel (2014). A nearly empty room was used for this task. The child sat on a chair with a desk. Candies and a bell were placed on the desk. The child was instructed as follows. The researcher will leave the 
room for 15 minutes (min). If the child does not eat the candies within this time, then more candies will be given. If the child does eat, then no more candies will be given. The child must not leave the chair. If the child perceives the condition unbearable, then the bell should be rung. After confirming the child's understanding, the researcher leaves the room. Brain activity was measured during the observation.

\subsection{Theory/Calculation}

In this study, I focused on the difference in the brain activity of the prefrontal cortex in EF between preschool children and lower-level children. The following hypotheses will be verified based on the knowledge obtained so far.

- There are differences between preschool children and lower-level school children siblings who share similar circumstances. Brain activity becomes more stable as one grows.

- Moreover, differences also occur in the results of the indicators for each subcomponent. The more difficult the subcomponent is, the more active the brain becomes, while its characteristics vary little with age.

The former means that EF may grow at some age. In particular, it means that the development is remarkable from early childhood to later childhood. Further, EF cannot be said to have grown if the brain activity is simply increased. Because it is apparent that it develops due to maturation, the corresponding weakening of brain activity means that it can be regarded as growth. In the latter, there is a task for each subcomponent, but the difficulty level differs for each task; thus, it is necessary to prepare a task that matches the development of each subcomponent. Because brain activity increases with the degree of difficulty, it is necessary to create a brain activity support suitable for children. Therefore, these can be assumed to be the hypotheses that could possibly predict the actual situation of EF in children and could possibly provide the appropriate support.

The following methods have been adopted to investigate this.

This study used functional near-infrared spectroscopy to measure brain activity in the prefrontal cortex during the performance of a representative index task of each subcomponent on EFs. In this regard, whether differences in brain activity can be observed between two siblings aged four $(4 ; 7-4 ; 8)$ and eight years $(8 ; 6-8 ; 7)$ as representatives of subgroups of children were investigated. The EFs of the DCCS task for cognitive shifts, the Stroop task for working memory, and the Marshmallow test for inhibitory functions were used for analysis. The brain activity (total- $\mathrm{Hb}$ in the left and right prefrontal cortex) in each task and the legitimate numbers were measured; the average value of each task is calculated. The means are compared, and a test of the difference of the means (t-test) is calculated using SPSS.

In other words, if the method is possible, the results of EF can be collected scientifically in real time and have considerable effectiveness in supporting EF in children.

\section{Results}

In terms of EF, brain activity in response to the representative index task of each subcomponent is measured in a cross-sectional manner using fNIRS. The study hypothesized that assessing the characteristics of the response activities of the prefrontal cortex is possible. The following tables present the results of the tasks of both the subjects (A and B).

Table 1 displays the amount of total- $\mathrm{Hb}(\mathrm{mMmm})$ for the left and right prefrontal cortex and the duration of each task for Subjects A and B. Table 2 presents the mean values for total-Hb (mMmm) for the left and right prefrontal cortex of each task for Subjects A and B. Table 3 shows the results of the t-test of the difference in the average values of each task for Subjects A and B.

For Subject A, significant differences were observed between the left and right prefrontal cortex in many tasks. That is, the study suggests that each task points to specific characteristics.

For Subject B, the results show that the WISC-IV task and Marshmallow test are more active than other indicators as regards brain activity. Furthermore, Subjects A and B show high levels of brain activity in the order of increasing difficulty in terms of behavioral indicators, as shown by the results. This finding is in agreement with that of other studies on neurophysiology, that is, brain activity becomes increasingly active as the task becomes increasingly difficult. 
Table 1. The amount of total-Hb (mMmm) for the left and right prefrontal cortex and the duration of each task for Subjects A and B

Subject A.

\begin{tabular}{lllll}
\hline Task & \multicolumn{2}{l}{ Total-Hb $(\mathrm{mMmm})$} & Time $(\mathrm{s})$ & $\begin{array}{l}\text { Behavioral evidence } \\
\text { Number of correct responses (each task)/ } \\
\end{array}$ \\
& Left & Right & & \begin{tabular}{l} 
waited time (Marshmallow test) (min) \\
\hline Stroop's task
\end{tabular} \\
& 0.166116358 & 0.199102714 & 60 & $14 / 15$ \\
& 0.150447512 & 0.288313201 & 60 & $15 / 15$ \\
& 0.213995247 & 0.195296959 & 60 & $15 / 15$ \\
DCCS_post & 0.189475849 & 0.21059055 & 60 & $15 / 15$ \\
& 0.376988676 & 0.382592353 & 80 & $6 / 6$ \\
& 0.312605684 & 0.293510497 & 76 & $6 / 6$ \\
& 0.290305516 & 0.258879224 & 100 & $6 / 6$ \\
DCCS_mixture & 0.848533309 & 0.403050544 & 97 & $6 / 6$ \\
& 1.063213459 & 0.919855311 & 341 & $8 / 12$ \\
& 0.941732666 & 0.538632103 & 200 & $12 / 12$ \\
& 0.221834619 & 0.325119066 & 135 & $12 / 12$ \\
WISC-IV & 0.904760241 & 0.634898291 & 199 & $12 / 12$ \\
& 2.056408705 & 1.081030546 & 759 & - \\
& 1.292535623 & 0.744593424 & 497 & - \\
Marshmallow & 0.279488085 & 0.325961747 & 437 & - \\
& 1.331071145 & 0.647139195 & 360 & 15 \\
& 1.709184579 & 1.123427818 & 900 & 15 \\
& 1.683160528 & 0.707878709 & 910 & 15
\end{tabular}

Note. Except for Stroop's task and combined DCCS, Subject A passed all tests. Based on this result, the study infers that the difficulty of the tasks was appropriate.

Subject $B$.

\begin{tabular}{lllll}
\hline Task & \multicolumn{2}{l}{ Total-Hb (mMm) } & & $\begin{array}{l}\text { Number of correct responses } \\
\text { (each task)/ } \\
\text { waited time (Marshmallow Test) (min) }\end{array}$ \\
\hline Stroop's task & 0.069899232 & 0.173472019 & 60 & $15 / 15$ \\
& 0.098004071 & 0.104997133 & 60 & $15 / 15$ \\
& 0.163476547 & 0.203471417 & 60 & $15 / 15$ \\
DCCS_post & 0.086321887 & 0.150997917 & 60 & $15 / 15$ \\
& 0.182968861 & 0.31664231 & 77 & $6 / 6$ \\
DCCS_mixture & 0.136417592 & 0.101399657 & 67 & $6 / 6$ \\
& 0.083034396 & 0.169713143 & 46 & $6 / 6$ \\
& 0.175802451 & 0.229662534 & 206 & $12 / 12$ \\
WISC-IV & 0.15680227 & 0.16933878 & 152 & $12 / 12$ \\
& 0.190913709 & 0.432042724 & 144 & $12 / 12$ \\
& 0.283078257 & 0.378114864 & 1102 & \\
Marshmallow & 0.558185197 & 0.567977183 & 719 & \\
& 0.239622238 & 0.550402901 & 682 & \\
& 0.614543056 & 0.753365427 & 900 & 15 \\
& 0.890247099 & 0.403904295 & 960 & 15 \\
& 0.449845677 & 0.476690076 & 950 & 15 \\
\hline
\end{tabular}

Note. Subject B passed all tests. 
Table 2. The mean values for total-Hb (mMmm) for the left and right frontal precortex of each task for Subjects $\mathrm{A}$ and $\mathrm{B}$

Subject $A$.

Subject $B$.

\begin{tabular}{lll}
\hline & \multicolumn{2}{l}{ Average Total-Hb (mMmm) } \\
\hline Task & Left & Right \\
Stroop's task & 0.184639536 & 0.231400237 \\
DCCS_post & 0.457108296 & 0.334508155 \\
DCCS_mixture & 0.782885246 & 0.604626193 \\
WISC-IV & 1.209477471 & 0.717195239 \\
Marshmallow & 1.574472084 & 0.826148574 \\
\hline
\end{tabular}

\begin{tabular}{lll}
\hline & \multicolumn{2}{l}{ Average of Total-Hb (mMm) } \\
\hline Task & Left & Right \\
Stroop's task & 0.104425434 & 0.158234621 \\
DCCS_post & 0.134140283 & 0.19591837 \\
DCCS_mixture & 0.174506144 & 0.277014679 \\
WISC-IV & 0.360295231 & 0.498831649 \\
Marshmallow & 0.651545278 & 0.544653266 \\
\hline
\end{tabular}

Note. The order of brain activation is similar to that of Subject B.

Table 3. The mean values for total- $\mathrm{Hb}(\mathrm{mMmm})$ for the left and right prefrontal cortex of each task for Subjects $\mathrm{A}$ and $\mathrm{B}$

Subject $A$.

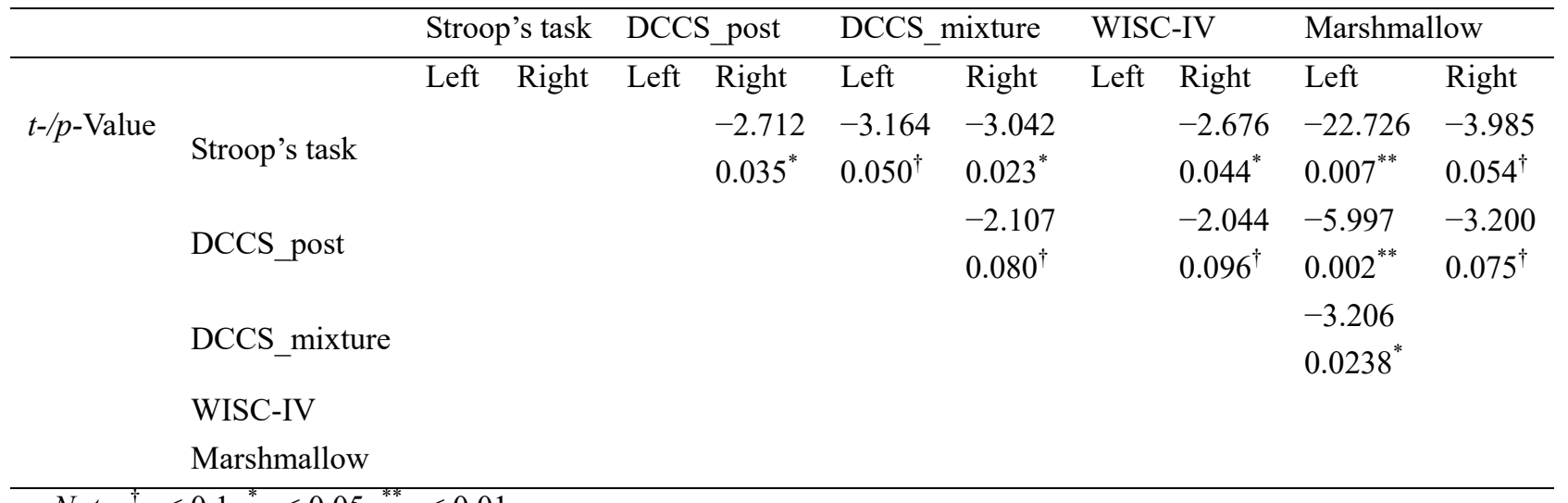

Note. ${ }^{\dagger} p<0.1 ;{ }^{*} p<0.05 ;{ }^{* *} p<0.01$

Subject $B$.

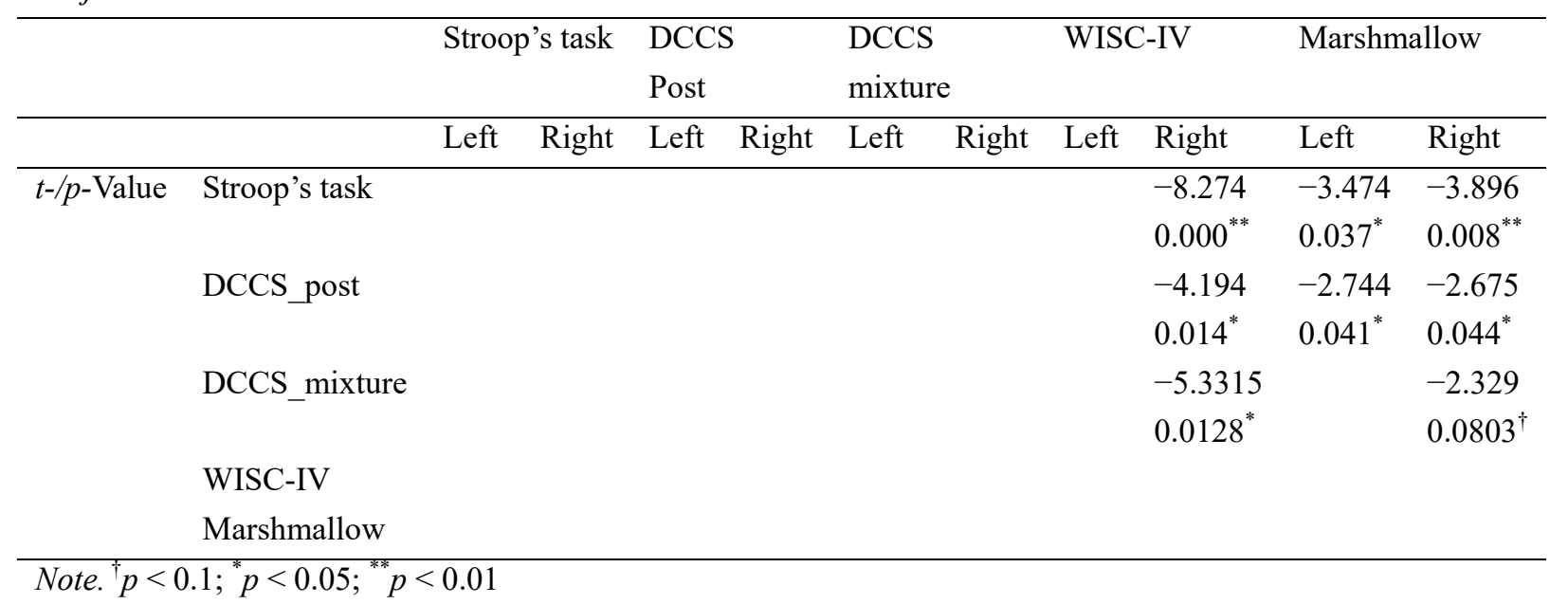


Based on the results, the study infers that assessing the characteristics of activity or reactions in the prefrontal cortex is possible by measuring the brain activity of the representative index task of each subcomponent using fNIRS.

The study then examines the hypothesis that such trends and characteristics of brain activity in the prefrontal cortex decrease with age and promotion of understanding.

Table 4 shows the results of the t-test of the difference between the average values of the same tasks for Subjects A and B. Significant differences were observed, except for WISC, in which the task differs according to age.

Subject A showed higher levels of reaction than Subject B, that is, brain activity stabilized with age and promotion of understanding. This finding is in agreement with that of neurophysiology, that is, cerebral activity is suppressed by stability associated with age and understanding.

Table 4. The results of the t-test of the difference between the average values of the same tasks for Subjects A and $\mathrm{B}$

\begin{tabular}{lllllll}
\hline Total-Hb $(\mathrm{mMmm})$ & $t$-Test & Stroop's task & $\begin{array}{l}\text { DCCS_ } \\
\text { post }\end{array}$ & $\begin{array}{l}\text { DCCS_ } \\
\text { mixture }\end{array}$ & WISC-IV & Marshmallow \\
\hline Left & t-Value & 3.052 & 2.047 & 2.704 & 1.62 & 5.211 \\
& $p$-Value & $0.022^{*}$ & $0.096^{\dagger}$ & $0.043^{*}$ & 0.181 & $0.006^{* *}$ \\
\multirow{2}{*}{ Right } & $t$-Value & 2.157 & 2.065 & 2.042 & 0.963 & 1.533 \\
& $p$-Value & $0.074^{\dagger}$ & $0.094^{\dagger}$ & $0.097^{\dagger}$ & 0.39 & 0.2 \\
\hline
\end{tabular}

${ }^{\dagger} p<0.1 ;{ }^{*} p<0.05 ;{ }^{* *} p<0.01$

\section{Discussion}

This study investigated the actual state of EF in early childhood, which is a higher-order cognitive control process and involved in cognitive development. By definition, EF primarily pertains to cognitive ability (Blair \& Razza, 2007; Bull, Espy, Wiebe, Espy, \& Charak, 2008; Espy et al., 2004) and is the key to future success (Casey et al., 2011; Moffitt et al., 2011). Several subcomponents have been identified under EF, and tasks used as indicators have been proposed. Therefore, evidence for EF as regards behavioral levels is abundant (Homack \& Riccio, 2004; Mischel, 2014; Sepeta et al., 2017; Toplak, Sorge, Benoit, West, \& Stanovich, 2010; Zelazo, 2006).

Moreover, neurophysiological evidence in this regard is increasing. At present, the relationship with the prefrontal cortex is being clarified (Adleman et al., 2002; Durston et al., 2006; Houdé, Rossi, Lubin, \& Joliot, 2010; Li et al., 2010; Mischel, 2014; Moriguchi \& Hiraki, 2009; Schroeter et al., 2004). Studies using fNIRS pointed out that the prefrontal cortex may be more activated when difficulties of tasks increases and may be less activated with increasing age.

Therefore, as an exploratory case study, the brain activity of the representative index task of each subcomponent was measured in a cross-sectional manner using fNIRS in siblings aged four and eight years, thus ensuring similar genetic and environmental backgrounds. Furthermore, it investigated the possibility of assessing the characteristics of brain activity in the prefrontal cortex.

Behavioral evidence addressing issues in previous studies is presented as follows. For the emotive aspect (cool), Stroop's task of suppression can be performed easily even at the age of four years. Children aged 4-5 years can succeed in the DCCS post-switch task that measures cognitive shift; however, they can fail the DCCS mixture task. Conversely, children aged over 5 years can pass the DCCS combined task (Zelazo, 2006). For work memory, the WISC-IV task has a high degree of difficulty because the level of task increases according to age and degree of understanding, such that it should be carried out to the point where the child can no longer provide answers. In terms of cognition (hot), the Marshmallow test can be passed by approximately one-third of the four-year-old children and the majority of five-year-old and older children (Mischel, Ebbesen, \& Raskoff Zeiss, 1972).

The results of the brain measurements using fNIRS indicated that brain activity increased according to more the level of difficulty of the behavioral index in the preschooler as well as the child in lower elementary school. Namely, brain measurements can elucidate individual characteristics in terms of EF during childhood. From these things, it was theoretically possible to make assumptions, but empirically it can be set as a new hypothesis. In addition, significant differences were observed among the index tasks, which may lead to the following points. Inhibition, cognitive shifting, and working memory have been proposed as subcomponents of EF (Miyake \& 
Friedman, 2012). However, EFs related to early childhood have only one subcomponent (Hughes, Ensor, Wilson, \& Graham, 2009; Moriguchi, 2015; Wiebe et al., 2008). Previous studies have proposed that the subcomponents will gradually differentiate (Tucker-Drob, 2009). However, in the current study, using three elements is deemed appropriate compared with using one element due to the significant differences observed in many tasks. Thus, the need and possibility emerge for the creation of a new index task with low, medium, and high levels of difficulty for each subcomponent.

Furthermore, the characteristics of brain activity in the prefrontal cortex associated with age and promotion of understanding were examined by comparing the brain activity of the siblings. Results reveal that Subject B showed less activated than Subject A of the same tasks. This finding also points to the concept of automation of information processing theory, which posits that individuals can solve problems efficiently as they grow older without using the entirety of the prefrontal cortex (Siegler, 1991). In addition, this finding is consistent with the general knowledge in brain science that brain activity stabilizes by practice and proficiency with repeated tasks (Sugiya, 2011; Verner, Herrmann, Troche, Roebers, \& Rammsayer, 2013). In addition, the similar characteristic observed in both the subjects is consistent with that of a previous research suggesting that EF has a strong genetic element, and the influence of the parenting environment is strong (Anderson et al., 2009; Friedman et al., 2008).

Practical research on support has been conducted, and certain effects have been obtained in relation to EF as being strongly influenced by the child-rearing environment (Barker et al., 2014; Diamond, Barnett, Thomas, \& Munro, 2007; Klingberg, Forssberg, \& Westerberg, 2002). Therefore, it is an urgent issue to provide a support method for promoting EF in difficult situations and for difficult children. In this research, I found the following regarding a new hypothesis.

Regarding EF of early childhood, (1) brain activity in the prefrontal cortex during infancy increases as the task becomes more difficult, but (2) its activity declines and stabilizes as increasing ages. (3) Such an evaluation can be easily accomplished with the two-channel fNIRS. It can be said that these are indispensable viewpoints for supporting the promotion of EFs because there is a possibility that the evaluation can be done easily in any situation and that the viewpoint of evaluation can be found.

Therefore, a new hypothesis could be presented as a possible method of child support. This result is a useful suggestion that will broaden the perspectives of not only scholars in the fields of psychology, pedagogy, and neuroscience but also parents and those involved in child support. Furthermore, the results can be used as reference for advance research on this field and to broaden its scope.

However, one of the limitations of this study is that it is a case study. Case studies cannot be used to determine causation but to develop a novel hypothesis. As such, comprehensive data are required to render the results generalizable across fields, age, and backgrounds. However, as exploratory research intends to present novel findings, a case study is important as an initial step. In addition, fNIRS is limited to two channels and should be linked to other brain measurements. Moreover, considerable measures are further required for other brain measurements due to numerous physical difficulties. Another limitation is that the behavior index tasks were representative of the targeted subcomponents. Clearly, other tasks should be considered. However, a case study is sufficient as a first step for exploratory research to provide novel findings in terms of differences for each subcomponent.

\section{Conclusions}

In this study, fNIRS was used to measure brain activity in the prefrontal cortex when performing representative index tasks for each subcomponent. The participants were a preschooler (4 years old, $4 ; 7-4 ; 8)$ and his older sister ( 8 years old, 8;6-8;7); a similar parenting environment. I verified that the characteristics of brain activity in the prefrontal cortex can be evaluated by cross-sectional measurement.

As a result of the experiment, the preschoolers and the children in the lower grades of the elementary school showed higher brain activity in the order of increasing difficulty of the behavior index. The results are similar to that of previous studies on neurophysiology, with increasing brain activity as tasks become more difficult. Moreover, it was found that preschoolers showed higher responsiveness than children in lower grades of elementary school, and brain activity was stabilized with increasing age and promotion of understanding. This is consistent with the neurophysiological findings that brain activity is suppressed by stabilization associated with aging and promotion of understanding. Further, numerous subcomponent tasks were found to have a certain significant difference.

In summary, the following possibilities have been obtained from exploratory cases. 
EF, which is closely related to the cognitive aspect of brain function, can identify certain trends and characteristics from prefrontal cortex activity in response to the tasks associated with each subcomponent. These findings of possibilities are important in clarifying the cognitive structure of early childhood for their care from the perspective of cognition and will contribute to the development of each in this academic field.

\section{Acknowledgments}

This work was supported by JSPS KAKENHI under grant number 19K03127.

The author would like to thank Enago (www.enago.jp) for the English language review.

\section{References}

Adleman, N. E., Menon, V., Blasey, C. M., White, C. D., Warsofsky, I. S., Glover, G. H., \& Reiss, A. L. (2002). A developmental fMRI study of the Stroop color-word task. Neuroimage, 16(1), 61-75. https://doi.org/10.1006/nimg.2001.1046

Anderson, B., Storfer-Isser, A., Taylor, H. G., Rosen, C. L., \& Redline, S. (2009). Associations of executive function with sleepiness and sleep duration in adolescents. Pediatrics, 123(4), e701-e707. https://doi.org/10.1542/peds.2008-1182

Barker, J. E., Semenov, A. D., Michaelson, L., Provan, L. S., Snyder, H. R., \& Munakata, Y. (2014). Less-structured time in children's daily lives predicts self-directed executive functioning. Frontiers in Psychology, 5, 593. https://doi.org/10.3389/fpsyg.2014.00593

Blair, C., \& Razza, R. P. (2007). Relating effortful control, executive function, and false belief understanding to emerging math and literacy ability in kindergarten. Child Development, 78(2), 647-663. https://doi.org/10.1111/j.1467-8624.2007.01019.x

Bull, R., Espy, K. A., \& Wiebe, S. A. (2008). Short-term memory, working memory, and executive functioning in preschoolers: Longitudinal predictors of mathematical achievement at age 7 years. Developmental Neuropsychology, 33(3), 205-228. https://doi.org/10.1080/87565640801982312

Burgess, P. W., \& Stuss, D. T. (2017). Fifty years of prefrontal cortex research: Impact on assessment. Journal of $\begin{array}{llll}\text { the International Neuropsychological } & \text { Society, } & \text { 23(9-10), }\end{array}$ https://doi.org/10.1017/S1355617717000704

Buss, A. T., \& Spencer, J. P. (2018). Changes in frontal and posterior cortical activity underlie the early emergence of executive function. Developmental Science, 21(4), e12602. https://doi.org/10.1111/desc. 12602

Casey, B. J., Somerville, L. H., Gotlib, I. H., Ayduk, O., Franklin, N. T., Askren, M. K., ... Shoda, Y. (2011). Behavioral and neural correlates of delay of gratification 40 years later. Proceedings of the National Academy of Sciences, 108(36), 14998-15003. https://doi.org/10.1073/pnas.1108561108

Diamond, A., Barnett, W. S., Thomas, J., \& Munro, S. (2007). The early years-Preschool program improves cognitive control. Science, 318(5855), 1387-1388. https://doi.org/10.1126/science.1151148

Durston, S., Davidson, M. C., Tottenham, N., Galvan, A., Spicer, J., Fossella, J. A., \& Casey, B. J. (2006). A shift from diffuse to focal cortical activity with development. Developmental Science, 9(1), 1-8. https://doi.org/10.1111/j.1467-7687.2005.00454.x

Espy, K. A., McDiarmid, M. M., Cwik, M. F., Stalets, M. M., Hamby, A., \& Senn, T. E. (2004). The contribution of executive functions to emergent mathematic skills in preschool children. Developmental Neuropsychology, 26(1), 465-486. https://doi.org/10.1207/s15326942dn2601_6

Friedman, N. P., Miyake, A., Young, S. E., DeFries, J. C., Corley, R. P., \& Hewitt, J. K. (2008). Individual differences in executive functions are almost entirely genetic in origin. Journal of Experimental Psychology: General, 137(2), 201-225. https://doi.org/10.1037/0096-3445.137.2.201

Haida, M. (2002). Implication of a signal from brain optical topography. Medix, 36, 17-21. Retrieved from https://www.hitachi.co.jp/products/healthcare/products-support///contents/medix/pdf/vol36/P17-21.pdf

Homack, S., \& Riccio, C. A. (2004). A meta-analysis of the sensitivity and specificity of the Stroop color and word test with children. Archives of Clinical Neuropsychology, 19(6), 725-743. https://doi.org/10.1016/j.acn.2003.09.003 
Hoshi, S. (2005). Functional near-infrared spectroscopy: Limitations and potential. The Journal of Japanese College of Angiology, 45(2), 61-67. Retrieved from https://j-ca.org/wp/wp-content/uploads/2016/03/4502_10ken_so.pdf

Houdé, O., Rossi, S., Lubin, A., \& Joliot, M. (2010). Mapping numerical processing, reading, and executive functions in the developing brain: An fMRI meta-analysis of 52 studies including 842 children. Developmental Science, 13(6), 876-885. https://doi.org/10.1111/j.1467-7687.2009.00938.x

Hughes, C., Ensor, R., Wilson, A., \& Graham, A. (2009). Tracking executive function across the transition to school: A latent variable approach. Developmental Neuropsychology, 35(1), 20-36. https://doi.org/10.1080/87565640903325691

Igawa, M., Atsumi, Y., Takahashi, K., Shiotsuka, S., Hirasawa, H., Yamamoto, R., ... Koizumi, H. (2001). Activation of visual cortex in REM sleep measured by 24-channel NIRS imaging. Psychiatry and Clinical Neurosciences, 55(3), 187-188. https://doi.org/10.1046/j.1440-1819.2001.00819.x

Kerr-German, A. N., \& Buss, A. T. (2020). Exploring the neural basis of selective and flexible dimensional attention: An fNIRS study. Journal of Cognition and Development, 21(3), 313-325. https://doi.org/10.1080/15248372.2020.1760279

Klingberg, T., Forssberg, H., \& Westerberg, H. (2002). Training of working memory in children with ADHD. Journal of Clinical and Experimental Neuropsychology, 24(6), 781-791. https://doi.org/10.1076/jcen.24.6.781.8395

Li, X., Lu, Z. L., D’Argembeau, A., Ng, M., \& Bechara, A. (2010). The Iowa gambling task in fMRI images. Human Brain Mapping, 31(3), 410-423. https://doi.org/10.1002/hbm.20875

Mischel, W. (2014). The marshmallow test: Understanding self-control and how to master it. Random House.

Mischel, W., Ebbesen, E. B., \& Raskoff Zeiss, A. (1972). Cognitive and attentional mechanisms in delay of gratification. Journal of Personality and Social Psychology, 21(2), 204-218. https://doi.org/10.1037/h0032198

Miyake, A., \& Friedman, N. P. (2012). The nature and organization of individual differences in executive functions: Four general conclusions. Current Directions in Psychological Science, 21(1), 8-14. https://doi.org/10.1177/0963721411429458

Miyake, A., Friedman, N. P., Emerson, M. J., Witzki, A. H., Howerter, A., \& Wager, T. D. (2000). The unity and diversity of executive functions and their contributions to complex "frontal lobe" tasks: A latent variable analysis. Cognitive Psychology, 41(1), 49-100. https://doi.org/10.1006/cogp.1999.0734

Moffitt, T. E., Arseneault, L., Belsky, D., Dickson, N., Hancox, R. J., Harrington, H., ... Caspi, A. (2011). A gradient of childhood self-control predicts health, wealth, and public safety. Proceedings of the National Academy of Sciences, 108(7), 2693-2698. https://doi.org/10.1073/pnas.1010076108

Moriguchi, Y. (2015). Early development of executive function, its neural mechanism and interventions. Japanese Psychological Review, 58(1), 77-88. Retrieved from https://www.jstage.jst.go.jp/article/sjpr/58/1/58_77/_pdf

Moriguchi, Y. (2019). The power to control yourself. Kodansha Ltd.

Moriguchi, Y., \& Hiraki, K. (2009). Neural origin of cognitive shifting in young children. Proceedings of the National Academy of Sciences, 106(14), 6017-6021. https://doi.org/10.1073/pnas.0809747106

Moriguchi, Y., \& Hiraki, K. (2013). Prefrontal cortex and executive function in young children: A review of NIRS studies. Frontiers in Human Neuroscience, 7, 867. https://doi.org/10.3389/fnhum.2013.00867

Schroeter, M. L., Zysset, S., Wahl, M., \& von Cramon, D. Y. (2004). Prefrontal activation due to Stroop interference increases during development-An event-related fNIRS study. Neuroimage, 23(4), 1317-1325. https://doi.org/10.1016/j.neuroimage.2004.08.001

Sepeta, L. N., Casaletto, K. B., Terwilliger, V., Facella-Ervolini, J., Sady, M., Mayo, J., .. Berl, M. M. (2017). The role of executive functioning in memory performance in pediatric focal epilepsy. Epilepsia, 58(2), 300-310. https://doi.org/10.1111/epi.13637

Siegler, R. S. (1991). Children's thinking. Englewood Cliffs, NJ: Prentice-Hall, Inc.

Stroop, J. R. (1935). Studies of interference in serial verbal reactions. Journal of Experimental Psychology, 18(6), 643-662. https://doi.org/10.1037/h0054651 
Stuss, D. T., \& Alexander, M. P. (2000). Executive functions and the frontal lobes: a conceptual view. Psychological Research, 63(3-4), 289-298. https://doi.org/10.1007/s004269900007

Sugiya, R. (2011). Changes in test scores and cortical activation during the Miyake memory test. Rigakuryoho Kagaku, 26(4), 537-540. https://doi.org/10.1589/rika.26.537

Toplak, M. E., Sorge, G. B., Benoit, A., West, R. F., \& Stanovich, K. E. (2010). Decision-making and cognitive abilities: A review of associations between Iowa gambling task performance, executive functions, and intelligence. Clinical Psychology Review, 30(5), 562-581. https://doi.org/10.1016/j.cpr.2010.04.002

Tucker-Drob, E. M. (2009). Differentiation of cognitive abilities across the life span. Developmental Psychology, 45(4), 1097-1118. https://doi.org/10.1037/a0015864

Verner, M., Herrmann, M. J., Troche, S. J., Roebers, C. M., \& Rammsayer, T. H. (2013). Cortical oxygen consumption in mental arithmetic as a function of task difficulty: A near-infrared spectroscopy approach. Frontiers in Human Neuroscience, 7, 217. https://doi.org/10.3389/fnhum.2013.00217

Villringer, A., Planck, J., Hock, C., Schleinkofer, L., \& Dirnagl, U. (1993). Near-infrared spectroscopy (NIRS): A new tool to study hemodynamic changes during activation of brain function in human adults. Neuroscience Letters, 154(1-2), 101-104. https://doi.org/10.1016/0304-3940(93)90181-J

Wechsler, D. (2003). The Wechsler Intelligence Scale for Children-fourth edition. London: Pearson. https://doi.org/10.1037/t15174-000

Wiebe, S. A., Espy, K. A., \& Charak, D. (2008). Using confirmatory factor analysis to understand executive control in preschool children: I. Latent structure. Developmental Psychology, 44(2), 575-587. https://doi.org/10.1037/0012-1649.44.2.575

Zelazo, P. D. (2006). The dimensional change card sort (DCCS): A method of assessing executive function in children. Nature Protocols, 1, 297-301. https://doi.org/10.1038/nprot.2006.46

Zelazo, P. D., \& Carlson, S. M. (2012). Hot and cool executive function in childhood and adolescence: Development and plasticity. Child Development Perspectives, 6(4), 354-360. https://doi.org/10.1111/j.1750-8606.2012.00246.x

\section{Copyrights}

Copyright for this article is retained by the author(s), with first publication rights granted to the journal.

This is an open-access article distributed under the terms and conditions of the Creative Commons Attribution license (http://creativecommons.org/licenses/by/4.0/). 